NIH) National Institutes of Health

\title{
NATIONAL INSTITUTES OF HEALTH
}

Pathways to Prevention Workshop:

The Role of Opioids in the Treatment of Chronic Pain

September 29-30, 2014

EXECUTIVE SUMMARY

The National Institutes of Health (NIH) workshop is co-sponsored by the NIH Office of Disease Prevention (ODP), the NIH Pain Consortium, the National Institute on Drug Abuse, and the National Institute of Neurological

Disorders and Stroke. A multidisciplinary Working Group developed the workshop agenda, and an evidence report was prepared by an Evidence-based Practice Center through a contract with the Agency for Healthcare Research and Quality to facilitate the workshop discussion. During the 11/2-day workshop, invited experts discussed the body of evidence, and attendees had opportunities to provide comments during open discussion periods. After weighing evidence from the evidence report, expert presentations, and public comments, an unbiased, independent panel prepared this draft report, which identifies research gaps and future research priorities. This draft report was posted on the ODP website and public comments were accepted for 2 weeks. The final report was then released to coincide with publication in a peer-review journal.

\section{Introduction}

Chronic pain affects an estimated 100 million Americans, or one-third of the U.S. population, although estimates vary depending upon how pain is defined and assessed. Approximately 25 million people experience moderate to severe chronic pain with significant pain-related activity limitations and diminished quality of life. In addition to the burden of suffering, pain is the primary reason Americans are on disability. The societal costs of chronic pain are estimated at between $\$ 560$ and $\$ 630$ billion per year as a result of missed work days and medical expenses.

Although numerous treatments are available for treatment of chronic pain, an estimated 5 to 8 million Americans use opioids for long-term management of chronic pain. Moreover, workshop speakers presented data from numerous sources that indicate a dramatic increase in opioid prescriptions and use over the past 20 years. For example, the number of prescriptions for 
opioids written for pain treatment in 1991 was 76 million; in 2011, this number reached 219 million. This striking increase in opioid prescriptions has paralleled the increase in opioid overdoses and hospital admissions. In fact, hospital admissions for prescription painkillers have increased more than fivefold in the last two decades. Yet, evidence also indicates that 40 to $70 \%$ of people with chronic pain are not receiving proper medical treatment, with concerns for both over- and under-treatment of chronic pain. Together, the prevalence of chronic pain and the increasing use of opioids have created a "silent epidemic" of distress, disability, and danger to a large percentage of Americans. The overriding question is whether we, as a nation, are currently approaching chronic pain in the best possible manner that maximizes effectiveness and minimizes harm. Several workshop speakers indicated that $80 \%$ of all opioid prescriptions worldwide are written in the United States. This suggests, in part, that other countries have found different treatments for chronic pain.

On September 29-30, 2014, the National Institutes of Health (NIH) convened a Pathways to Prevention Workshop: The Role of Opioids in the Treatment of Chronic Pain. Specifically, the workshop addressed four key questions:

1. What is the long-term effectiveness of opioids?

2. What are the safety and harms of opioids in patients with chronic pain?

3. What are the effects of different opioid management strategies?

4. What is the effectiveness of risk mitigation strategies for opioid treatment?

To answer these questions, the Pacific Northwest Evidence-based Practice Center, under contract to the Agency for Healthcare Research and Quality, completed a review of the literature related to these questions. The NIH conducted a 11/2-day workshop featuring more than 20 speakers with 
varied expertise and viewpoints. In addition, audience members expressed many other experiences and views during the discussion periods.

\section{Context}

To understand the dilemma of opioids and chronic pain, the panel felt strongly that an understanding of underlying contextual factors was crucial. Many workshop presentations provided information about these contextual factors, including background on the scope of patient pain and its treatment, the patient's experience of pain and pain management, the current public health issues associated with treatment of pain, and the historical context that underlies the current use and overuse of opioids in the treatment of chronic pain.

As noted in the introduction, pain affects millions of Americans, and the societal costs are high. For patients, chronic pain is often associated with psychological distress, social disruptions, disability, and high medical expenses. In addition, with an increase in chronic pain, there has been a simultaneous rise in opioid use. This use has been associated with pain relief, but also with an increase in adverse outcomes (e.g., addiction, overdose, insufficient pain relief).

Given the rise in chronic pain syndromes and the poor outcomes associated with opioid treatment, the panel felt it was fundamental to understand the patient's perspective. At the workshop, the panel heard from individuals struggling with chronic pain as well as advocates for afflicted individuals. The burden of dealing with unremitting pain can be devastating to the patient's psychological well-being and can negatively affect a person's ability to maintain gainful employment or achieve meaningful advancement professionally. It can affect

relationships with spouses and significant others and may limit engagement with friends and 
other social activities. The prospect of living a lifetime with pain may induce fear and demoralization and can lead to diagnoses of anxiety and depression.

Coupled with psychological and social effects are the negative encounters that many individuals with chronic pain experience with the health care system. Health care providers, often poorly trained in management of chronic pain, are sometimes quick to label patients as "drug-seeking" or as "addicts" who overestimate their pain. Some doctors "fire" patients for increasing their dose or merely for continuing to voice concerns about their pain management. Some patients have had similarly negative interactions with pharmacists. These experiences may make patients feel stigmatized, or feel as if others view them as criminals. These experiences may heighten fears that pain-relieving medications will be "taken away," leaving the patient in chronic, disabling pain. In addition, negative perceptions by clinicians can create a rupture in the therapeutic alliance, which some studies have identified as impeding successful opioid treatment. For example, cultural factors may influence the treatment a patient receives from health care providers. White providers tend to underestimate the pain of black patients and perceive them to be at higher risk than white patients for substance abuse.

Biased media reports on opioids also affect patients. Stories that focus on opioid misuse and fatalities related to opioid overdose may increase anxiety and fear among some stable, treated patients that their medications could be tapered or discontinued to "prevent addiction." For example, one workshop presentation indicated that a typical news story about opioids was likely to exclude information about the legitimate prescription use of opioids for pain, focusing instead on overdose, addiction, and criminal activity. 
However, the panel also wanted to emphasize what was reflected in numerous presentations at the workshop: Many patients have been compliant with their prescriptions, and some feel that their pain is managed adequately to the point of satisfactory quality of life. However, many patients using opioids long-term continue to have moderate to severe pain and diminished quality of life. While many physicians feel that opioid treatment can be valuable for some patients, physicians also feel that patient expectations for pain relief may be unrealistic and that long-term opioid prescribing can complicate and impair their therapeutic alliance with the patient.

The patient perspective is incredibly important, and yet it is only one aspect of the problem. Another equally important consideration is how prescription opioids used in the treatment of chronic pain create public health problems. In other words, although some patients experience substantial pain relief from prescription opioids and do not suffer adverse effects, these benefits have to be weighed against problems caused by the vast number of opioids now prescribed.

According to the Centers for Disease Control and Prevention, there were approximately 17,000 overdose deaths involving opioids in 2011. From 2000 to 2010, the number of admissions for addiction to prescription opioids increased more than four-fold, to over 160,000 per year. Different age groups are affected differently. For example, in 2010, one out of eight deaths of 25- to 34-year-olds was opioid-related. There are also collateral deaths from those who have been prescribed opioids. In a 3-year period (2003 to 2006), more than 9,000 children were exposed to opioids; this figure is based on data from toxicology centers, and the actual number may be higher. Of these, nearly all children ingested the opioid (99\%), and the ingestion occurred in the home (92\%). A small number of children died (n=8), but 43 children suffered major effects, and 214 suffered moderate effects. Neonatal narcotic withdrawal also has 
increased, with an estimated 29,000 infants affected. Both short-term physiological problems as well as long-term behavioral consequences result from this withdrawal.

There is some concern that opioids are now becoming gateway drugs for heroin use. For example, one study found that among individuals with a heroin addiction in the 1960s, the most common first opioid used (the entry drug into heroin) was heroin itself. However, by the year 2000, the most common entry drug to heroin use was a prescription opioid.

Speakers at the workshop expressed almost unanimous concern that physicians are unable to distinguish among individuals who would use opioids for pain management and not develop problems with misuse, those who would use them for pain management and then become addicted, and those who request a prescription because of a primary substance use disorder. Only a minority of those who use opioids for chronic pain become addicted. For example, in one study of individuals treated for chronic pain, the addiction prevalence, depending on criteria, ranged from about 14 to $19 \%$.

Finally, there is a major public health concern that opioids are finding their way illicitly into the public arena. The Substance Abuse and Mental Health Services Administration's 2013 National Survey on Drug Use and Health found that, among people age 12 and older abusing analgesics, $53 \%$ reported receiving them for free from a friend or relative. Only $23.8 \%$ received prescriptions from one or more doctors.

Another key contextual factor the panel considered was a historical perspective. The panel identified important historical factors related to approval by the U.S. Food and Drug Administration (FDA) of opioid medications, introduction of new opioid medications (particularly extended-release formulations), training of prescribers, and health system changes. 
Different opioids have undergone varying levels of scrutiny by the FDA. All current, extendedrelease opioids have been approved for chronic pain based on 12-week adequate and wellcontrolled efficacy studies, although there are safety data for extended-release opioids for a year, in most cases from open-label studies. A number of immediate-release opioids had been on the market without prior approval; however, in recent years, all of them have received FDA approval for acute pain.

The introduction of new opioid drugs on the market over the past decade, particularly those with extended-release formulations, made them attractive to patients and clinicians who perceived them as safe and effective. As indicated above, there were limited long-term studies on which to base clinical decisions. Physicians have little training in how to manage chronic pain patients and did not have to demonstrate knowledge in how to prescribe these medications.

As described below in "Challenges Within the Health Care System," changes in the health care system have provided perverse incentives for clinicians to prescribe opioids in the brief amount of time they have with patients.

Of course, the historical and current context of opioid use and prescription is complicated by the heterogeneity of the problem. There are many facets of heterogeneity: patients (e.g., age, gender, race); the pain etiology (e.g., peripheral vs. central pain), diverse clinical presentations that include various comorbidities; characteristics of the clinical setting (e.g., providers, payment structures); and the available opioids for prescription (e.g., differential receptor affinities, pharmacokinetics, potential for drug interactions).

Given these complexities, the panel struggled with striking a balance between two ethical principles, beneficence and doing no harm, specifically between the clinically indicated 
prescribing of opioids on the one hand and the desire to prevent inappropriate prescription abuse and harmful outcomes on the other. These goals should not be mutually exclusive and, in fact, approaches that attempt to achieve both goals simultaneously are essential to move the field of chronic pain management forward. However, one of the central struggles the panel grappled with in making recommendations is the dearth of empirical evidence to support the four key questions addressed by the Evidence-based Practice Center (EPC) report. Thus, in order to formulate the recommendations in this report, the panel synthesized both the evidence from the EPC report and the workshop presentations that focused on clinical experience as well as on smaller trials and cohort studies (e.g., non-randomized clinical trials).

\section{Clinical Issues}

\section{Patient Assessment and Triage}

Chronic pain is a complex clinical issue requiring an individualized, multifaceted approach. Contributing to the complexity is the fact that chronic pain is not limited to a particular disease state but rather spans a multitude of conditions, with varied etiologies and presentations. Yet, traditionally, persons living with chronic pain are "lumped" into a single category, and treatment approaches have been generalized with little evidence to support this practice. In addition, although pain is a dynamic phenomenon, waxing and waning and changing in nature over time, it is often viewed and managed with a static approach. For a number of reasons-including lack of knowledge, practice settings, resource availability, and reimbursement structure-clinicians are often ill-prepared to diagnose, appropriately assess, treat, and monitor patients with chronic pain. Based on the evidence report and the workshop presentations, the panel has identified several clinical management issues worthy of further discussion. 
First, there must be recognition that patients' manifestation of and response to pain is varied, with genetic, cultural, and psychosocial factors all contributing to this variation. Evidence was presented that clinicians' response to patients with pain also differs, often due to preconceived notions and biases based on racial, ethnic, and other sociodemographic stereotypes. The totality of the data points to the need for an individualized, patient-centered approach based on a biopsychosocial model as opposed to the biomedical model that is more commonly employed. Treating pain and reducing suffering do not always equate, and many times patients and clinicians have disparate ideas about successful outcomes. A more holistic approach to the management of chronic pain, inclusive of the patients' perspectives and desired outcomes, should be the goal.

Patients, providers, and advocates all agree that there is a subset of patients for whom opioids are an effective treatment method for their chronic pain, and that limiting or denying access to opioids for these patients can be harmful. It appears that these patients can be safely monitored using a structured approach, which includes optimization of opioid therapy, management of adverse effects, and brief follow-up visits at regular intervals. Therefore, recommendations regarding the clinical use of opioids should avoid disruptive and potentially harmful changes in patients currently benefiting from this treatment.

This concept - that some patients benefit while others may receive no benefit or in fact may be harmed - highlights the current challenges of appropriate patient selection. Data are lacking on accuracy of and effectiveness of risk prediction instruments for identifying patients at highest risk for the development of adverse outcomes (e.g., overdose, development of an opioid use disorder). Yet, longitudinal studies have demonstrated risk factors (e.g., substance use disorders, other comorbid psychiatric illnesses) that are more likely to be associated with these harmful 
outcomes, and some studies show that patients who are at high risk are most likely to be prescribed opioids (and higher doses of opioids). Ideally, patients with these risk factors would be less likely to receive opioids or more likely to receive them in the context of a maximally structured approach; however, studies of large clinical databases suggest the opposite.

Although the literature to support use of specific risk assessment tools is insufficient, the consensus appears to be that the approach to the management of chronic pain should be individualized, based on a comprehensive clinical assessment that is conducted with dignity and respect and without value judgments or stigmatization of the patient. Based on the workshop presentations, this initial evaluation would include an appraisal of pain intensity, functional status, and quality of life, as well as an assessment of known risk factors for potential harm, including history of substance use disorders and current substance use; presence of mood, stress, or anxiety disorders; medical comorbidity; and concurrent use of medications with potential drug-drug interactions. Additionally, there may be a role for the redesign of the electronic health record to facilitate such an assessment, including integration of meaningful use criteria to increase its adoption. Finally, incorporating the use of other clinical tools (e.g., prescription drug monitoring programs) into this assessment, although not well studied, seems to be widely supported. These factors also can be used to tailor the clinical approach, triaging those screening at highest risk for harm to more structured and higher intensity monitoring approaches.

\section{Treatment Options}

Despite what is commonly done in current clinical practice, there appear to be few data to support the long-term use of opioids for chronic pain management. Several workshop speakers stressed the need to use treatment options that include a range of progressive approaches that 
might initially include nonpharmacological options, such as physical therapy, behavioral therapy, and/or proven complementary and alternative medicine approaches with demonstrated efficacy, followed by pharmacological options, including non-opioid pharmacotherapies. The use of and progression through these treatment modalities would be guided by the patient's underlying disease state, pain, and risk profile as well as clinical and functional status and progress. However, according to a workshop speaker, lack of knowledge or limited availability of these nonpharmacological modalities and the ready availability of pharmacological options and associated reimbursement structure appear to steer clinicians towards the use of pharmacological treatment and, more specifically, opioids.

One area of clinical importance the panel reviewed was the notion that pain type could influence pain management. Data were presented on three distinct pain mechanisms: (1) peripheral nociceptive — caused by tissue damage or inflammation, (2) peripheral neuropathic — damage or dysfunction of peripheral nerves, and (3) centralized—characterized by a disturbance in the processing of pain by the brain and spinal cord. Individuals with more peripheral/nociceptive types of pain (e.g., acute pain due to injury, rheumatoid arthritis, cancer pain) may respond better to opioid analgesics. In contrast, those with central pain syndromes-exemplified by fibromyalgia, irritable bowel syndrome, temporal-mandibular joint disease and tension headache — do not respond as well to opioids, as to centrally acting neuroactive compounds (e.g., certain antidepressant medications, anticonvulsants). In particular, there is strong evidence for non-opioid interventions in treatment of fibromyalgia, one of the most common conditions presenting in primary care and pain clinics. In fact, the workshop presented interesting preliminary evidence that if an initial evaluation for pain demonstrated even a few signs of fibromyalgia (not meeting criteria for the full syndrome), the patient was at risk for poor 
response to opioids and a worse long-term course of pain. In addition, speakers presented evidence that nearly all chronic pain may have a centralized component, and it was suggested that opioids may promote progression from acute nociceptive pain to chronic centralized pain. However, several speakers and audience members cautioned against making blanket statements about who is or is not likely to benefit from opioids, again highlighting the importance of individualized patient assessment and management. The health care system would benefit from additional research on these different mechanisms of pain and the optimal approaches for each, including the value of individualized versus general approaches; identifying risk factors for patients most likely to develop chronic pain after an acute or subacute pain syndrome, and ways to mitigate or reduce the risk of transitioning to a chronic pain syndrome.

\section{Clinical Management}

There is little evidence to guide clinicians once they have made the decision to initiate opioids for chronic pain therapy. Data on selection of specific agents based on opioid characteristics, dosing strategies, and titration or tapering of opioids are insufficient to guide current clinical practice. Discussed during the workshop was the concept of opioid rotation, in which a clinician transitions a patient from an existing opioid regimen to another with the goal of improving therapeutic outcomes. However, this approach has not been formally evaluated. The use of equianalgesic tables (opioid conversion tables), which provide a list of equianalgesic doses of various opioids to guide clinicians in determining doses for converting from one opioid to another, was an issue of particular concern. The equianalgesic dose is a construct based on estimates of relative opioid potency. A multitude of these opioid conversion tables are available in both the peer-reviewed and non-peer-reviewed literature, and speakers noted the lack of consistency between the tables. Many of the studies to determine these equianalgesic doses were 
conducted in a sample of the study population and using data points that may not generalize to patients presenting with chronic pain. The FDA has begun including data obtained from drug trials and post-marketing studies in package inserts to aid clinicians in switching between opioids, but it appears that many clinicians and pharmacists are not aware of this. Furthermore, although three known classes of opioid receptors - mu $(\mu)$, kappa $(\kappa)$, and delta $(\Delta)$ - have been identified, multiple receptor subtypes within each of these classes can alter the effect of opioids based on receptor subtype binding. This led to a discussion between workshop speakers of the concept of incomplete cross-tolerance, in which providers may need to reduce the dose by 25 to $30 \%$ when converting between one opioid and another. Because of its longer half-life, methadone may require a larger reduction (up to 90\%); in fact, some speakers argued that methadone should be excluded from these tables and suggested that the use of these tables may have led to harm and should not be broadly used. There was a call for the development of validated and patient-specific types of equianalgesic tables. It was also noted that the majority of clinicians receive little to no education on use of these tables and on converting from one opioid regimen to another; this should be a focus of future clinical education and clinical decision support efforts.

\section{Determination and Assessment of Outcomes}

Several workshop speakers noted that patient assessments should be ongoing, including both positive and negative outcomes. The range of items on such assessments might include pain intensity and pain frequency, using both a short time reference as well as a longer timeframe for comparative purposes, functional status including impact on functions of daily living; quality of life; depression; anxiety; potential misuse or abuse of opioid medications; potential adverse medical effects of opioids; and other measures that mimic those items obtained during the initial 
clinical risk profiling. These frequent reassessments should guide maintenance or modification of the current treatment regimen, and patients who are failing to meet the mutually agreed upon clinical outcomes should be considered for discontinuation of opioid therapy. Although there appeared to be consensus among speakers on the need for an "exit strategy," there was less consensus and very few data on how one should be implemented.

\section{Adverse Events and Side Effects}

In addition to the very real risk of development of an opioid use disorder, chronic administration of opioids are associated with other adverse effects, including increased risk of falls and fractures, hypogonadism with resultant sexual dysfunction, and, in at least two studies, increased risk of myocardial infarction. These factors are important to the discussion of risks versus benefits with patients, and realistic expectations regarding adverse events and side effects from various treatment options may need to be explained to patients as well as relatives and home care providers. New patient communication options may be of value for the patients or relatives to discuss evolving concerns. Adverse events and side effects might be monitored regularly and reported to the clinician between regularly scheduled visits using web or other communication channels.

\section{Risk Mitigation Strategies}

As with much of the other data on opioid use for chronic pain, data are limited on the efficacy of various risk mitigation strategies, including patient agreements, urine drug screening, and pill counts. Some speakers expressed concern as to the effectiveness of patient agreements as few data are available to support their use. However, the use of patient agreements and other care support mechanisms might be an option as part of a comprehensive care management plan and 
might be reinforced without the use of judgmental perspectives that could impact the relationship between patient and provider. Naloxone, which traditionally has been used to reverse heroin overdose, was highlighted as a potential risk mitigation strategy for patients who are prescribed opioids for chronic pain. Guided by the premise that these are risky drugs as opposed to risky patients, a workshop speaker suggested that naloxone might be provided to patients at the same time as the original prescription for the opioid and that this might provide an opportunity for additional patient education. Other speakers were more cautious about using this strategy for all patients, yet were willing to consider that it might be explored from an individual patient riskbenefit perspective.

\section{Reducing the Next Generation of Chronic Opioid Users}

Speakers stated that a multidisciplinary team approach that emulates the functions of a multidisciplinary pain clinic would be desirable, given the prior history of success of such models in treating the whole person and not merely the pain condition, which may not be a simple, single entity. As noted above, different types of pain-peripheral nocioceptive, peripheral neuropathic, and centralized pain - appear to have different profiles of response to such treatments. Furthermore, the use of a more effective chronic disease care model may have implications for reducing the potential of a new generation of chronic opioid users as the continued first-line use of opioids for chronic pain treatment is generally suboptimal and has the potential for addiction. Although the team composition may vary, members might include the primary care provider, case or care managers, nurses, pharmacists, psychologists, psychiatrists, social workers, and other pain specialists. However, the current health care financial incentives do not appear to bode well for the re-initiation of such an approach (see below for a more detailed discussion of this point). Finally, one simple approach the panel considered to decrease 
the conversion of acute users to chronic users was to advise those prescribing opioid medications for the treatment of acute pain (e.g., in the post-operative setting or for an injury) to prescribe fewer pills to be taken over a shorter but clinically reasonable timeframe, as there is some evidence that higher numbers of pills initially prescribed is related to risk of chronicity of use.

\section{Challenges Within the Health Care System}

A major influence on opioid prescribing is the evolution of the larger health care system and the current state of primary care. The panel heard reports of major problems with the current health care system, including:

- Poor support for team-based care and specialty pain clinics

- Over-burdened primary care providers

- A lack of knowledge and decision support for chronic pain management

- Financial misalignment favoring the use of medications

- Fragmentation of care across different providers.

Pain is a multidimensional problem ranging from discomfort to agony and affecting physical, emotional, and cognitive function as well as interpersonal relationships and social roles. As with other chronic conditions, chronic pain management requires a more comprehensive biopsychosocial model of care. Therefore, best practice models for chronic pain management require a multidisciplinary approach similar to that recommended for other chronic complex illnesses such as depression, dementia, eating disorders, or diabetes. Research demonstrates that these conditions can be managed successfully using an interdisciplinary team-based approach to care (e.g., medicine, psychology, nursing, pharmacy, social work). Early efforts to manage pain in the late 20th century were based on similar effective models of interdisciplinary, 
comprehensive, and individualized care. Unfortunately, as health care systems evolved and increasingly implemented and maintained only those interventions that were declared to be revenue-generating, team-based approaches to care for pain were largely abandoned.

Instead, management of chronic pain has been largely relegated to the primary care providers working in health systems not designed or equipped for chronic pain management. Moreover, expectations for primary care providers increasingly evolved towards productivity-based metrics, with more tasks expected to be completed within a 10- to 20-minute office visit. Primary care providers often face competing clinical priorities in patients with chronic pain because these patients often have multi-morbidity and polypharmacy. Administrative responsibilities also compete for the provider's time. For example, growing requirements for documentation in the electronic health record are consuming a larger portion of the office visit. Hence, timeconsuming but important clinical tasks—such as conducting multidimensional assessments, developing personalized care plans, and counseling — have given way to care processes that can be accomplished more quickly and with fewer resources, such as prescription writing and referrals. In the case of pain management, which often takes substantial face-to-face time, quicker alternatives have become the default option. As a result, providers often prescribe opioids for pain even when, for any given patient, the pain might be treated more safely and effectively with other modalities.

Primary care providers are charged with relieving pain as a professional obligation and a fundamental goal of health care. However, these providers have often received little specific training in chronic pain management or in the use and management of opioids. This may be particularly true for those providers who were trained before newer formulations of opioids or other alternatives were available. As the systematic review clearly reveals, these providers do not 
have access to evidence-based dosing schedules, adjustment and switching rules, or tapering and stopping rules to guide pain management. Even if primary care providers had the requisite knowledge, skill, and intent, they often do not have access to the resources needed to manage pain according to current guidelines. This is often true because alternative first-line treatment strategies are not available. For example, most practices do not have access to experts in pain management, including specialty pain clinics, or access to the alternative approaches to pain management (e.g., physical therapy, cognitive and behavioral approaches, acupuncture, yoga, meditation, other complementary and alternative medicine). Therefore, clinicians provide a prescription for opioids because they and their patients feel it is the only or the most expedient alternative. Once the decision to initiate opioids has been made, patients and providers lack practical tools to monitor the outcomes of chronic pain management. For example, simple monitoring tools (e.g., the Patient Health Questionnaire-9 for depression) assist in the diagnosis and management of depression. Although widely available, pain rating scales alone are not comprehensive enough to measure the adequacy of pain control on important dimensions such as quality of life, function, and employment.

Payment structures and incentives also represent an important system-level facilitator for excessive opioid use. Fee-for-service payment traditionally has not focused on the outcomes of care valued by patients, but rather on the processes of medical care. Current reimbursement for evaluation and management may be inadequate to reflect the time and team-based approaches needed for integrative treatment. In some instances, payment structures place barriers to nonopioid therapy, such as formulary restrictions that require evidence of failure of multiple therapies before covering non-opioid alternatives (e.g., pregabalin). Other payment structures, such as tiered coverage systems, keep non-opioid alternatives as second- or third-line options 
rather than placing them more appropriately as first-line therapy. Other incentives encourage prescribing opioids for several months at a time rather than for a shorter term or using lowervolume prescriptions, because providers are instructed that patient and administrative costs are lower, and that convenience is improved with longer-duration and larger-volume prescriptions. The panel heard reports that this apparently benign incentive actually may lead to an increased risk of opioid dependence or other adverse events, including harm through nonmedical uses. Moreover, current reimbursement policies do not provide payment for some of the health professionals who are needed to provide best-practice pain management (e.g., pharmacists, care coordinators) and others may be reimbursed insufficiently (e.g., for only a single visit). In health systems that are primarily fee-for-services, there may be incentives to generate short-term revenue, whereas in capitated systems, where physicians receive a set amount for each enrolled person per given period of time, there may be greater incentive to invest in upfront resources (e.g., team-based care) if they can prevent downstream utilization (e.g., hospitalization). Given the current vagaries of payment structures, perhaps it is not surprising that providers and patients chose opioids more often than is clinically appropriate and more often than guidelines suggest.

Finally, fragmentation of care across multiple providers and sites of care often leads to patients receiving prescriptions from multiple providers. This may lead not only to inappropriate prescribing of opioids but also to inappropriate prescribing of unsafe combinations of drugs such as opioids and benzodiazepines. Up to $25 \%$ of patients who have chronic pain receive their medications in the emergency department, often effectively bypassing the primary care system. Patients with chronic pain may see multiple specialists with relevant expertise in chronic pain (e.g., neurologists, orthopedists, rheumatologists, psychiatrists), but these specialists may often prescribe opioids without the knowledge of primary care providers. The specialists may focus on 
pain in isolation and may not recognize or consider the patient's comorbid conditions, concomitant medications, or goals of care. Patients may actively "shop" for providers (within or across health care systems or state lines) to find a provider who is willing to prescribe opioids.

The panel heard recommendations that there is a clear need to address these system-level problems. Chief among these recommendations is the need to develop, evaluate, and implement new models of care for chronic pain management. To accomplish this fundamental goal, research must address health care aims and thus assess the costs and benefits to individuals and populations. Moving to team-based care is unlikely to happen without restructuring reimbursement systems, building patient-centered clinical information systems, expanding the roles and responsibilities of health care professionals beyond the physician, and conducting new basic research on which patients require which care in which settings.

\section{Methods and Measurement}

Reliable and valid clinical and research methods are essential as the medical field seeks to understand best practices for chronic pain management. The EPC report found few long-term (more than 1 year) studies of opioid treatment, and those identified in the literature were typically of poor quality (see Summary of Findings Table). It is particularly difficult to extrapolate from studies examining the effects of opioids on acute pain to chronic pain. The panel identified methodological problems related to definitions, measurement, and research design.

Definitions. One of the central definitional problems is defining acute versus chronic pain. Various durations are used to define chronic pain, including "lasting more than 3 months" or "lasting more than 6 months" or a time-based definition that is somewhat arbitrary. For example, 
the American Academy of Pain Medicine suggests that chronic pain is best defined as pain that does not remit in the expected amount of time. This is clearly an individualized pain assessment and, although it may be useful to the individual clinician, does not provide a standard definition that could be used for research purposes. The panel suggested that detectable changes in brain function occur as pain moves from acute to chronic states; however, although this may provide a more precise, functional definition of pain, it is unrealistic to expect that most research will incorporate neuroimaging modalities.

Unclear definitions also impair the understanding of the types of pain that patients experience. Many research studies compare patients with cancer-derived and non-cancer-derived pain. This dichotomy is clearly insufficient, as neither cancer pain nor non-cancer pain are homogeneous, in large part because individual differences in sensory processing and augmented pain affect perception and reporting of pain. In other words, chronic pain is heterogeneous and complex. As mentioned previously, one workshop presentation classified pain by its source: peripheral (nociceptive) pain, peripheral (non-nociceptive) pain, and centralized. Although this rubric may be useful for considerations of acute pain, chronic pain should not be partitioned into mutually exclusive, discrete categories. This definitional problem affects diagnosis, treatment, and drug regulation.

Finally, definitions are important when considering how to measure outcomes. Pain relief is a major focus of treatment and research. However, it is difficult to quantify pain. The typically used 0-10 pain scale provides an overall sense of pain, but not an assessment of individual components related to pain. For example, recent work on the concept of "fibromyalgianess" (the tendency to respond to illness and psychosocial stress with fatigue, widespread pain, general increase in symptoms, and similar factors) identifies at least three components to chronic pain 
that are important to measure: chronic pain or irritation in specific body regions, somatic symptoms (e.g., fatigue, sleep, mood, memory), and sensitivity to sensory stimuli.

Measurement. Research also suffers from significant measurement problems. Risk screening instruments would help clinicians implement better risk management strategies. Many speakers at the workshop indicated that the field does not have good risk assessment tools. The EPC report found that standardized tools lacked sufficient sensitivity and specificity to make them clinically useful. In large part, the problem with screening is that it is not clear what risk factors should be measured or whether it is feasible or sensible to screen for risk. Some speakers indicated that clinicians should assume that all patients are at risk and not use valuable resources (including clinician time) to screen.

Finally, patient outcomes (typically measured in an ongoing manner) are important. Numerous speakers indicated that the primary goal for researchers and clinicians may be the reduction in patient pain; however, patients may be more interested in improving quality of life, rather than absolute pain reduction. Functional behavior related to pain also needs to be assessed.

The most important aspect of measuring patient outcomes is to acknowledge that they are determined by multiple factors and therefore will need to be multidimensional in scope. Key components of a thorough assessment of patient outcomes would include measures of pain, psychopathology, quality of life, social factors (e.g., days worked), safety, and adverse outcomes.

Research Design. The panel reviewed several presentations related to study design. Based on the EPC report, there is a clear need for well-designed longitudinal studies of effectiveness and safety of long-term opioid use in the management of chronic pain; this is an immediate concern. Such studies - both because of their length and the heterogeneity of factors to be accounted for- 
would need to be large and would therefore be expensive. In addition, it is not clear from a practical standpoint that patients with chronic pain would be willing to be randomized to placebo, nonpharmacological treatments, or non-opioid medications. The workshop speakers also proposed an alternative design, which involved accepting patients on long-term treatment into a study and randomizing them to maintenance versus tapering of the opioid. However, speakers noted similar practical issues around recruitment of individuals willing to have their medication tapered. Pragmatic designs (with some flexibility in the treatments used) could help bypass some of the challenges with conducting long-term randomized control trials.

With these limitations, workshop speakers suggested other types of longitudinal studies; for example, an approach using a small cohort study was seen as a more feasible option. Also from a feasibility standpoint, the use of the electronic health record to track pain and markers of improvement as well as adverse outcomes and side effects may provide the best data on large populations. In addition, some speakers noted limitations of FDA-mandated post-marketing surveillance studies by pharmaceutical companies, but also saw this as an opportunity to gain valuable information in this area.

Another design issue considered by the panel related to how best to account for heterogeneity across patients, medications, and outcomes. Novel design and statistical approaches may be needed to manage this complexity. For example, ecological designs that embrace heterogeneity and help to understand diversity among patients and to identify key subgroups that may respond differently to various treatments should be considered. This methodology often incorporates novel statistical methods (e.g., latent class and profile analyses). 
The panel also noted several specific research issues that merit further exploration. These include the following:

1. Better understanding is needed of the window between effective dose and dose at which side effects and adverse outcomes occur. These may include studies on how this window is defined and assessed as well as the drug-related, genetic, and other patient-related factors that might affect the targeted dose range.

2. In adverse outcomes research, it is important to determine how best to model more immediate versus longer-term side effects based on the length of exposure to opioids. The notion was presented that some poor outcomes (e.g., falls) might occur soon after initiation of treatment, whereas others (e.g., hypogonadism) might be more associated with longer-term exposure. Future studies will need to encompass this time-varying aspect of certain adverse effects and poor outcomes.

3. Few studies have looked at genetic predictors of response and poor outcomes. There are several promising areas and specific loci for genetic research in this area, including a panel of gene variants related to cytochrome P450 metabolism (e.g., examining outcomes in people who are slow, intermediate, or fast drug metabolizers), receptor target single nucleotide polymorphisms (SNPs) as well as SNPs related to indirect modulation (e.g., COMT, the gene coding for catechol-O-mehtyltransferase), the drug transporter (e.g., $A B C B 1$ ), and other polymorphisms derived from genome-wide association studies (e.g., rs2952768).

Incorporation of biological approaches will be important to gaining a better understanding of the etiology of chronic pain and the mechanisms involved in opiate response and poor outcomes. 
Greater incorporation of functional imaging studies of pain, as well as clinical neuroscience findings about salient psychological factors, hold promise for identifying patients who would best respond to opioids versus other pharmacological or nonpharmacological modalities. Finally, future studies might examine the utility of variables such as evoked pain sensitivity and endogenous opioid activity.

Implementation science may be useful to address some of the clinical and practice issues. For example, research on how to bring prescription monitoring systems into an electronic health record may be particularly important. Finding ways to incorporate pharmacists and nurses in care groups is also essential. A final example includes research into the cost-effectiveness of chronic pain management teams, particularly given potential incentives for providing high quality of care (e.g., pay for performance).

\section{Complementary Efforts}

As the medical community looks for ways to increase available options to control pain and suffering, many complementary groups are at work. A 2011 report by the Institute of Medicine, Relieving Pain in America: A Blueprint for Transforming Prevention, Care, Education, and Research, has sparked efforts from various agencies to partner in addressing this issue.

The NIH Pain Consortium has selected 12 health professional schools as Centers of Excellence in Pain Education (CoEPEs). The CoEPEs will act as hubs for the development, evaluation, and distribution of pain management curriculum resources for medical, dental, nursing, and pharmacy schools to enhance and improve how health care professionals are taught about pain and its treatment. 
The Stanford-NIH Pain Registry, now called the National Collaborative Health Outcomes Information Registry (CHOIR) system, provides clinicians with valuable information regarding treatment outcomes. This platform collects outcome data on large numbers of patients suffering from chronic pain.

The Interagency Pain Research Coordinating Committee is a federal advisory committee charged with coordination of all pain research efforts across all federal agencies. The ultimate goal of the committee is to advance the fundamental understanding of pain and to improve pain-related treatment strategies.

The FDA has recognized that extended-release and long-acting opioids are associated with serious risks. The FDA is now requiring additional studies and clinical trials to assess these risks, which include misuse, abuse, hyperalgesia, addiction, overdose, and death.

Many professional societies have taken a stance on the use of opioids for chronic pain. The American Academy of Neurology recently published a position paper on non-cancer pain. Initiatives such as the American Board of Internal Medicine Foundation's "Choosing Wisely" are underway.

\section{Summary of EPC Report Findings}

\section{Effectiveness and comparative effectiveness}

a. In patients with chronic pain, what is the effectiveness of long-term opioid therapy for longterm ( $\geq 1$ year) outcomes related to pain, function, and quality of life? 


\begin{tabular}{|l|l|l|}
\hline Key Question & $\begin{array}{l}\text { Strength of } \\
\text { Evidence }\end{array}$ & Conclusion \\
\hline Pain, function, quality of life & Insufficient & $\begin{array}{l}\text { No study of opioid therapy versus placebo } \\
\text { or no opioid therapy evaluated long-term } \\
\text { ( } \geq 1 \text { year) outcomes related to pain, } \\
\text { function, or quality of life. }\end{array}$ \\
\hline
\end{tabular}

\section{Harms and adverse events}

a. In patients with chronic pain, what are the risks of opioids versus placebo or no opioid on

(1) opioid abuse, addiction, and related outcomes; (2) overdose; and (3) other harms?

\begin{tabular}{|c|c|c|}
\hline Key Question & $\begin{array}{l}\text { Strength of } \\
\text { Evidence }\end{array}$ & Conclusion \\
\hline Abuse, addiction & Low & $\begin{array}{l}\text { No randomized trial was evaluated. One } \\
\text { retrospective cohort study found prescribed } \\
\text { long-term opioid use was associated with } \\
\text { significantly increased risk of abuse or } \\
\text { dependence versus no opioid use. }\end{array}$ \\
\hline Abuse, addiction & Insufficient & $\begin{array}{l}\text { In } 10 \text { uncontrolled studies, estimates of } \\
\text { opioid abuse, addiction, and related } \\
\text { outcomes varied substantially even after } \\
\text { stratification by clinic setting. }\end{array}$ \\
\hline Overdose & Low & $\begin{array}{l}\text { Current opioid use was associated with } \\
\text { increased risk of any overdose events } \\
\text { (adjusted HR 5.2, 95\% CI } 2.1 \text { to 12) and } \\
\text { serious overdose events (adjusted HR 8.4, } \\
95 \% \text { CI } 2.5 \text { to 28) versus current nonuse. }\end{array}$ \\
\hline Fractures & Low & $\begin{array}{l}\text { Opioid use was associated with increased } \\
\text { risk of fracture in one cohort study } \\
\text { (adjusted HR } 1.28,95 \% \text { CI } 0.99 \text { to } 1.64 \text { ) } \\
\text { and one case-control study (adjusted OR } \\
1.27,95 \% \text { CI } 1.21 \text { to } 1.33 \text { ). }\end{array}$ \\
\hline Myocardial infarction & Low & $\begin{array}{l}\text { Current opioid use was associated with } \\
\text { increased risk of myocardial infarction } \\
\text { versus nonuse (adjusted OR } 1.28,95 \% \text { CI } \\
1.19 \text { to } 1.37 \text { and incidence rate ratio } 2.66 \text {, } \\
95 \% \text { CI } 2.30 \text { to } 3.08 \text { ). }\end{array}$ \\
\hline Endocrine & Low & $\begin{array}{l}\text { Long-term opioid use was associated with } \\
\text { increased risk of use of medications for } \\
\text { erectile dysfunction or testosterone } \\
\text { replacement versus nonuse (adjusted OR } \\
1.5,95 \% \text { CI } 1.1 \text { to } 1.9 \text { ). }\end{array}$ \\
\hline
\end{tabular}


b. How do harms vary depending on the dose of opioids used?

\begin{tabular}{|c|c|c|}
\hline Key Question & $\begin{array}{l}\text { Strength of } \\
\text { Evidence }\end{array}$ & Conclusion \\
\hline Abuse, addiction & Low & $\begin{array}{l}\text { One retrospective cohort study found } \\
\text { higher doses of long-term opioid therapy } \\
\text { associated with increased risk of opioid } \\
\text { abuse or dependence than lower doses. } \\
\text { Compared to no opioid prescription, the } \\
\text { adjusted odds ratios were } 15 \text { ( } 95 \% \text { CI } 10 \text { to } \\
21 \text { ) for } 1-36 \mathrm{MED} / \text { day, } 29 \text { (95\% CI } 20 \text { to } \\
41 \text { ) for } 36-120 \mathrm{MED} / \text { day, and } 122 \text { (95\% } \\
\text { CI } 73 \text { to } 205 \text { ) for } \geq 120 \mathrm{MED/day.}\end{array}$ \\
\hline Overdose & Low & $\begin{array}{l}\text { Versus } 1 \text { to } 19 \mathrm{mg} \text { MED/day, one cohort } \\
\text { study found an adjusted HR for an } \\
\text { overdose event of } 1.44 \text { ( } 95 \% \text { CI } 0.57 \text { to } \\
3.62 \text { ) for } 20 \text { to } 49 \mathrm{mg} \text { MED/day and } 11.18 \\
\text { (95\% CI } 4.80 \text { to } 26.03 \text { ) at }>100 \mathrm{mg} \\
\text { MED/day; one case-control study found an } \\
\text { adjusted OR for an opioid-related death of } \\
1.32 \text { (95\% CI } 0.94 \text { to } 1.84 \text { ) for } 20 \text { to } 49 \mathrm{mg} \\
\text { MED/day and } 2.88 \text { (95\% CI } 1.79 \text { to } 4.63) \\
\text { at } \geq 200 \mathrm{mg} \text { MED/day. }\end{array}$ \\
\hline Fracture & Low & $\begin{array}{l}\text { Risk of fracture increased from an adjusted } \\
\mathrm{HR} \text { of } 1.20 \text { (95\% CI } 0.92 \text { to } 1.56) \text { at } 1 \text { to } \\
<20 \mathrm{mg} \mathrm{MED} / \mathrm{day} \text { to } 2.00 \text { ( } 95 \% \mathrm{CI} 1.24 \text { to } \\
3.24) \text { at } \geq 50 \mathrm{mg} \mathrm{MED} / \text { day; the trend was } \\
\text { of borderline statistical significance. }\end{array}$ \\
\hline Myocardial infarction & Low & $\begin{array}{l}\text { Relative to a cumulative dose of } 0 \text { to } 1350 \\
\text { mg MED over } 90 \text { days, the incidence rate } \\
\text { ratio for myocardial infarction for } 1350 \text { to } \\
<2700 \mathrm{mg} \text { was } 1.21 \text { ( } 95 \% \text { CI } 1.02 \text { to } 1.45 \text { ), } \\
\text { for } 2700 \text { to }<8100 \mathrm{mg} \text { was } 1.42 \text { ( } 95 \% \text { CI } \\
1.21 \text { to } 1.67 \text { ), for } 8100 \text { to }<18,000 \mathrm{mg} \text { was } \\
1.89(95 \% \text { CI } 1.54 \text { to } 2.33 \text { ), and for } \\
>18,000 \mathrm{mg} \text { was } 1.73 \text { (95\% CI } 1.32 \text { to } \\
2.26 \text { ). }\end{array}$ \\
\hline Motor vehicle accidents & Low & $\begin{array}{l}\text { No association was found between opioid } \\
\text { dose and risk of motor vehicle accidents. }\end{array}$ \\
\hline Endocrine & Low & $\begin{array}{l}\text { Relative to } 0 \text { to }<20 \mathrm{mg} \text { MED/day, the } \\
\text { adjusted OR for daily opioid dose of } \geq 120 \\
\text { mg MED/day for use of medications for } \\
\text { erectile dysfunction or testosterone } \\
\text { replacement was } 1.6 \text { ( } 95 \% \text { CI } 1.0 \text { to } 2.4) \text {. }\end{array}$ \\
\hline
\end{tabular}




\section{Dosing strategies}

a. In patients with chronic pain, what is the comparative effectiveness of different methods for initiating and titrating opioids for outcomes and risk?

\begin{tabular}{|l|l|l|}
\hline Key Question & $\begin{array}{l}\text { Strength of } \\
\text { Evidence }\end{array}$ & Conclusion \\
\hline Pain & Insufficient & $\begin{array}{l}\text { Evidence from three trials on effects of } \\
\text { titration with immediate-release versus } \\
\text { sustained-release opioids reported } \\
\text { inconsistent results on outcomes related to } \\
\text { pain. }\end{array}$ \\
\hline
\end{tabular}

c. In patients with chronic pain, what is the comparative effectiveness of different long-acting opioids on outcomes related to pain, function, and quality of life as well as the risk of overdose, addiction, abuse, or misuse?

\begin{tabular}{|l|l|l|}
\hline Key Question & $\begin{array}{l}\text { Strength of } \\
\text { Evidence }\end{array}$ & Conclusion \\
\hline Pain and function & Low & $\begin{array}{l}\text { No difference was found between various } \\
\text { long-acting opioids. }\end{array}$ \\
\hline $\begin{array}{l}\text { Assessment of risk of overdose, } \\
\text { addiction, abuse, or misuse }\end{array}$ & Insufficient & $\begin{array}{l}\text { No studies were designed to assess risk of } \\
\text { overdose, addiction, abuse, or misuse. }\end{array}$ \\
\hline $\begin{array}{l}\text { Overdose (as indicated by all- } \\
\text { cause mortality) }\end{array}$ & Low & $\begin{array}{l}\text { One cohort study found methadone to be } \\
\text { associated with lower all-cause mortality } \\
\text { risk than sustained-release morphine in a } \\
\text { propensity-adjusted analysis. }\end{array}$ \\
\hline Abuse and related outcomes & Insufficient & $\begin{array}{l}\text { One cohort study found some differences } \\
\text { between long-acting opioids in rates of } \\
\text { adverse outcomes related to abuse, but } \\
\text { outcomes were nonspecific for opioid- } \\
\text { related adverse events, precluding reliable } \\
\text { conclusions. }\end{array}$ \\
\hline
\end{tabular}


f. In patients with chronic pain on long-term opioid therapy, what is the comparative effectiveness of dose escalation versus dose maintenance or use of dose thresholds on outcomes related to pain, function, and quality of life?

\begin{tabular}{|l|l|l|}
\hline Key Question & $\begin{array}{l}\text { Strength of } \\
\text { Evidence }\end{array}$ & Conclusion \\
\hline $\begin{array}{l}\text { Pain, function, withdrawal due } \\
\text { to opioid misuse }\end{array}$ & Low & $\begin{array}{l}\text { No difference was found between more } \\
\text { liberal dose escalation versus maintenance } \\
\text { of current doses in pain, function, or risk of } \\
\text { withdrawal due to opioid misuse, but there } \\
\text { was limited separation in opioid doses } \\
\text { between groups (52 vs. } 40 \mathrm{mg} \text { MED/day at } \\
\text { the end of the trial). }\end{array}$ \\
\hline
\end{tabular}

h. In patients on long-term opioid therapy, what is the comparative effectiveness of different strategies for treating acute exacerbations of chronic pain on outcomes related to pain, function, and quality of life?

\begin{tabular}{|l|l|l|}
\hline Key Question & $\begin{array}{l}\text { Strength of } \\
\text { Evidence }\end{array}$ & Conclusion \\
\hline Pain & Moderate & $\begin{array}{l}\text { Two randomized trials found buccal } \\
\text { fentanyl more effective than placebo for } \\
\text { treating acute exacerbations of pain, and } \\
\end{array}$ \\
& $\begin{array}{l}\text { three randomized trials found buccal } \\
\text { fentanyl or intranasal fentanyl more } \\
\text { effective than oral opioids for treating } \\
\text { acute exacerbations of pain in patients on } \\
\text { long-term opioid therapy. }\end{array}$ \\
\hline
\end{tabular}


i. In patients on long-term opioid therapy, what are the effects of decreasing opioid doses or tapering off opioids versus continuation of opioids on outcomes related to pain, function, quality of life, and withdrawal?

\begin{tabular}{|l|l|l|}
\hline Key Question & $\begin{array}{l}\text { Strength of } \\
\text { Evidence }\end{array}$ & Conclusion \\
\hline Pain, function & Insufficient & $\begin{array}{l}\text { Abrupt cessation of morphine was } \\
\text { associated with increased pain and } \\
\text { decreased function compared to } \\
\text { continuation of morphine. }\end{array}$ \\
\hline
\end{tabular}

j. In patients on long-term opioid therapy, what is the comparative effectiveness of different tapering protocols and strategies on measures related to pain, function, quality of life, withdrawal symptoms, and likelihood of opioid cessation?

\begin{tabular}{|l|l|l|}
\hline Key Question & $\begin{array}{l}\text { Strength of } \\
\text { Evidence }\end{array}$ & Conclusion \\
\hline Opioid abstinence & Insufficient & $\begin{array}{l}\text { No clear differences were found between } \\
\text { different methods for opioid } \\
\text { discontinuation or tapering in likelihood of } \\
\text { opioid abstinence after 3 to 6 months. }\end{array}$ \\
\hline
\end{tabular}

\section{Risk assessment and risk mitigation strategies}

a. In patients with chronic pain being considered for long-term opioid therapy, what is the accuracy of instruments for predicting risk of opioid overdose, addiction, abuse, or misuse?

\begin{tabular}{|c|c|c|}
\hline Key Question & $\begin{array}{l}\text { Strength of } \\
\text { Evidence }\end{array}$ & Conclusion \\
\hline $\begin{array}{l}\text { Diagnostic accuracy: Opioid } \\
\text { Risk Tool }\end{array}$ & Insufficient & $\begin{array}{l}\text { Based on a cutoff of }>4 \text {, three studies (all } \\
\text { poor quality) reported very inconsistent } \\
\text { estimates of diagnostic accuracy, } \\
\text { precluding reliable conclusions. }\end{array}$ \\
\hline $\begin{array}{l}\text { Diagnostic accuracy: Screening } \\
\text { and Opioid Assessment for } \\
\text { Patients with Pain (SOAPP) } \\
\text { version } 1\end{array}$ & Low & $\begin{array}{l}\text { Based on a cutoff score of } \geq 8, \text { sensitivity } \\
\text { was } 0.68 \text { and specificity of } 0.38 \text { in one } \\
\text { study, for a PLR of } 1.11 \text { and NLR of } 0.83 \\
\text { Based on a cutoff score of }>6 \text {, sensitivity } \\
\text { was } 0.73 \text { in one study. }\end{array}$ \\
\hline
\end{tabular}


b. In patients with chronic pain, what is the effectiveness of use of risk prediction instruments on outcomes related to overdose, addiction, abuse, or misuse?

\begin{tabular}{|l|l|l|}
\hline Key Question & $\begin{array}{l}\text { Strength of } \\
\text { Evidence }\end{array}$ & Conclusion \\
\hline Outcomes related to abuse & Insufficient & $\begin{array}{l}\text { No study evaluated the effectiveness of } \\
\text { risk prediction instruments for reducing } \\
\text { outcomes related to overdose, addiction, } \\
\text { abuse, or misuse. }\end{array}$ \\
\hline
\end{tabular}

Abbreviations: $\mathrm{CI}=$ confidence interval, $\mathrm{HR}=$ hazard ratio, $\mathrm{MED}=$ morphine equivalent dose, $\mathrm{mg}=$ milligrams, $\mathrm{NLR}=$ negative likelihood ratio, $\mathrm{OR}=$ odds ratio, $\mathrm{PLR}=$ positive likelihood ratio

As can be seen in the above table, the EPC found a paucity of studies on the long-term (more than 1 year) outcomes of opioid treatment for chronic pain, and those identified in the literature were typically of poor quality. Further, there are insufficient data to guide appropriate patient assessment, opioid selection, dosing strategies, or risk mitigation. This underscores the need for high-quality research that focuses on establishing the appropriateness of long-term opioid treatment for the management of chronic pain. After listening to workshop speakers and audience members and examining the limited availability of studies on long-term opioid treatment, the panel makes the following recommendations:

1. Federal and non-federal agencies should sponsor research to identify which types of pain, specific diseases, and patients are most likely to benefit and incur harm from opioids. Such studies could use a range of study approaches and could include demographic, psychological, socio-cultural, ecological, and biological characterizations of patients in combinations with clear and accepted definitions of chronic pain and well-characterized records for opioid and other pain medications. 
2. Federal and non-federal agencies should sponsor the development and evaluation of multidisciplinary pain interventions, including cost-benefit analyses and identification of barriers to dissemination.

3. Federal and non-federal agencies should sponsor research to develop and validate research measurement tools for identification of patient risk and outcomes (including benefit and harm) related to long-term opioid use that can be adapted for clinical settings.

4. Electronic health record vendors and health systems should incorporate decision support for pain management and facilitate export of clinical data to be combined with data from other health systems to better identify patients who benefit from or are harmed by opioid use.

5. Researchers on the effectiveness and harm of opioids should consider alternative designs (e.g., $\mathrm{N}$ of 1 trials, qualitative studies, implementation science, secondary analysis, Phase 1 and 2 design) in addition to randomized clinical trials.

6. Federal and non-federal agencies should sponsor research on risk identification and mitigation strategies, including drug monitoring, prior to widespread integration of these into clinical care. This research should also assess how policy initiatives impact patient/public health outcomes.

7. Federal and non-federal agencies and health care systems should sponsor research and quality improvement efforts to facilitate evidence-based decision-making at every step of the clinical decision process.

8. In the absence of definitive evidence, clinicians and health care systems should follow current guidelines by professional societies about which patients and which types of pain 
should be treated with opioids, and about how best to monitor patients and mitigate risk for harm.

9. NIH or other federal agencies should sponsor conferences to promote harmonization of guidelines of professional organizations to facilitate their implementation more consistently in clinical care.

\section{Summary}

The rise in the number of Americans with chronic pain and the concurrent increase in the use of opioids to treat this pain have created a situation where large numbers of Americans are receiving suboptimal care. Patients who are in pain are often denied the most effective comprehensive treatments; conversely, many patients are inappropriately prescribed medications that may be ineffective and potentially harmful. At the root of the problem is the inadequate knowledge about the best approaches to treat various types of pain, balancing the effectiveness with the potential for harm, as well as a dysfunctional health care delivery system that encourages clinicians to prescribe the easiest rather than the best approach for addressing pain. The EPC report identified few studies that were able to answer the key questions, suggesting the dire need for research on the effectiveness and safety of opioids as well as optimal management and risk mitigation strategies. What was particularly striking to the panel was the realization that there is insufficient evidence for every clinical decision that a provider needs to make regarding the use of opioids for chronic pain, leaving the provider to rely on his or her own clinical experience. 
Because of the inherent difficulties of studying pain and the large number of patients already receiving opioids, new research designs and analytic methods will be needed to adequately answer the important clinical and research questions.

Until the needed research is conducted, health care delivery systems and clinicians must rely on the existing evidence as well as guidelines issued by professional societies, which need to be continually updated and harmonized to reflect recent research evidence and changes in expert opinion. Systems of care must facilitate the implementation of these guidelines rather than relying solely on individual clinicians, who are often overburdened and have insufficient resources.

Clearly, there are some patients for whom opioids are the best treatment for their chronic pain. However, for many more, there are likely to be more effective approaches. The challenge is to identify the conditions in patients for which opioid use is most appropriate, the regimens that are optimal, the alternatives for those who are unlikely to benefit from opioids, and the best approach to ensuring that every patient's individual needs are met by a patient-centered health care system. For the more than 100 million Americans living with chronic pain, meeting this challenge cannot wait. 


\title{
National Institutes of Health Office of Disease Prevention \\ Pathways to Prevention Workshop: \\ The Role of Opioids in the Treatment of Chronic Pain
}

September 29 - 30, 2014

\section{Panelists}

\author{
David B. Reuben, M.D., Panel Chairperson \\ Chief \\ Division of Geriatrics \\ Director \\ Multicampus Program in Geriatric Medicine and \\ Gerontology \\ Professor of Medicine \\ Division of Geriatrics \\ David Geffen School of Medicine at the University of \\ California, Los Angeles
}

Anika A. H. Alvanzo, M.D., M.S.

Assistant Professor of Medicine

Medical Director

Substance Use Disorders Consultation

Service

Johns Hopkins University School of Medicine

Baltimore, Maryland

Takamaru Ashikaga, Ph.D.

Director

Biometry Facility

College of Medicine

University of Vermont

Burlington, Vermont

G. Anne Bogat, Ph.D.

Professor

Department of Psychology

Michigan State University

East Lansing, Michigan
Christopher M. Callahan, M.D.

Professor of Medicine

Cornelius and Yvonne Pettinga Professor

Indiana University (IU) School of Medicine

Director and Research Scientist

IU Center for Aging Research

Research Scientist

Regenstrief Institute, Inc.

Indianapolis, Indiana

Victoria Ruffing, R.N., CCRC

Director of Patient Education

Director of Nursing

Johns Hopkins University Arthritis Center

Johns Hopkins School of Medicine

Adjunct Faculty

Johns Hopkins University School of Nursing

Baltimore, Maryland

David C. Steffens, M.D., M.H.S.

Professor and Chair

Department of Psychiatry

University of Connecticut Health Center

Farmington, Connecticut 


\section{Speakers}

Jane C. Ballantyne, M.D.

Professor, Retired

Department of Anesthesiology and Pain

Medicine

University of Washington

Philadelphia, Pennsylvania

Stephen Bruehl, Ph.D.

Professor

Department of Anesthesiology

Vanderbilt University School of Medicine

Nashville, Tennessee

Roger Chou, M.D., FACP

Director

Pacific Northwest Evidence-based Practice Center

Professor of Medicine

Department of Medical Informatics \&

Clinical Epidemiology

Oregon Health \& Science University

Portland, Oregon

Myra Christopher, L.H.D. (Hon.)

Kathleen M. Foley Chair for Pain and

Palliative Care

Center for Practical Bioethics

Kansas City, Missouri

Daniel J. Clauw, M.D.

Professor of Anesthesiology, Medicine and

Psychiatry

Director

Chronic Pain and Fatigue Research Center University of Michigan Medical Center

Ann Arbor, Michigan
Phillip O. Coffin, M.D., M.I.A.

Director of Substance Use Research

HIV Prevention Section

San Francisco Department of Public Health

San Francisco, California

Wilson M. Compton, M.D., M.P.E.

Deputy Director

National Institute on Drug Abuse

National Institutes of Health

Bethesda, Maryland

Edward C. Covington, M.D.

Director

Neurological Center for Pain

Cleveland Clinic

Cleveland, Ohio

Ricardo A. Cruciani, M.D., Ph.D.

Director

Center for Comprehensive Pain

Management and Palliative Care

Capital Institute for Neuroscience

Capital Health Medical Center

Hopewell, New Jersey

Tracy W. Gaudet, M.D.

Director

Office of Patient Centered Care and Cultural

Transformation

Veterans Health Administration

U.S. Department of Veterans Affairs

Washington, DC

Joseph T. Hanlon, Pharm.D., M.S.

Professor

Division of Geriatrics/Gerontology

Department of Medicine

University of Pittsburgh School of Medicine

Pittsburgh, Pennsylvania 
Nathaniel P. Katz, M.D., M.S.

President and Chief Executive Officer

Analgesic Solutions

Adjunct Assistant Professor of Anesthesia

Tufts University School of Medicine

Natick, Massachusetts

Erin E. Krebs, M.D., M.P.H.

Core Investigator

Center for Chronic Disease Outcomes

Research

Medical Director

Women Veterans Comprehensive Health

Center

Minneapolis Veterans Administration

Associate Professor

Department of Medicine

University of Minnesota

Minneapolis, Minnesota

Story C. Landis, Ph.D.

Director

National Institute of Neurological Disorders and Stroke

National Institutes of Health

Bethesda, Maryland

Jane C. Maxwell, Ph.D.

Senior Research Scientist

Addiction Research Institute

The University of Texas at Austin School of

Social Work

Austin, Texas

David M. Murray, Ph.D.

Associate Director for Prevention

Director

Office of Disease Prevention

Division of Program Coordination,

Planning, and Strategic Initiatives

Office of the Director

National Institutes of Health

Bethesda, Maryland
Steven D. Passik, Ph.D.

Vice President

Clinical Research and Advocacy

Millenium Research Institute

Millennium Laboratories

San Diego, California

Russell K. Portenoy, M.D.

Chief Medical Officer

Metropolitan Jewish Health System (MJHS)

Hospice and Palliative Care

Director

MJHS Institute for Innovation in Palliative Care

Professor of Neurology

Albert Einstein College of Medicine

New York, New York

David B. Reuben, M.D.

Chief

Division of Geriatrics

Director

Multicampus Program in Geriatric Medicine and Gerontology

Professor of Medicine

Division of Geriatrics

David Geffen School of Medicine at the University of California, Los Angeles

Los Angeles, California

Wendy B. Smith, Ph.D., M.A., BCB

Senior Scientific Advisor for Research

Development and Outreach

Office of Behavioral and Social Sciences

Research

Office of the Director

National Institutes of Health

Bethesda, Maryland

David J. Tauben, M.D.

Clinical Associate Professor

Department of Medicine

Chief (Interim)

Division of Pain Medicine

Medical Director

Center for Pain Relief

University of Washington

Seattle, Washington 
David A. Thomas, Ph.D.

Deputy Director

Division of Clinical Neurosciences and

Behavioral Research

National Institute on Drug Abuse

National Institutes of Health

Bethesda, Maryland

Judith Turner, Ph.D.

Professor

Department of Psychiatry and Behavioral Sciences

University of Washington

Seattle, Washington
Nora D. Volkow, M.D.

Director

National Institute on Drug Abuse

National Institutes of Health

Bethesda, Maryland

Sharon Walsh, Ph.D.

Professor of Behavioral Sciences, Psychiatry, Pharmacology, and Pharmaceutical Sciences

Director

Center on Drug and Alcohol Research

University of Kentucky College of Medicine

Lexington, Kentucky 


\section{Working Group Planning Meeting for the National Institutes of Health (NIH) Pathways to Prevention Workshop: Effectiveness and Risks of Long-Term Opioid Treatment of Chronic Pain}

August 28-29, 2013

\section{Working Group}

Chairpersons: $\quad$ David A. Thomas, Ph.D.

Deputy Director

Division of Clinical Neurosciences and Behavioral Research

National Institute on Drug Abuse

National Institutes of Health

Richard A. Denisco, M.D., Ph.D.

Services Research Branch

National Institute on Drug Abuse

National Institutes of Health

Caroline Acker, Ph.D.

Associate Professor and Head

Department of History

Carnegie Mellon University

Pittsburgh, Pennsylvania

Jane C. Ballantyne, M.D.

Professor, Retired

Department of Anesthesiology and Pain

Medicine

University of Washington

Philadelphia, Pennsylvania

Wen G. Chen, Ph.D.

Program Director

Sensory and Motor Disorders of Aging

Behavioral and Systems Neuroscience

Branch

Division of Neuroscience

National Institute on Aging

National Institutes of Health

Bethesda, Maryland

Edward C. Covington, M.D.

Director

Neurological Center for Pain

Cleveland Clinic

Cleveland, Ohio
Jody Engel, M.A., R.D.

Director of Communications

Office of Disease Prevention

Division of Program Coordination, Planning, and Strategic Initiatives

Office of the Director

National Institutes of Health

Bethesda, Maryland

Roger B. Fillingim, Ph.D.

Professor

University of Florida College of Dentistry

Director

University of Florida Pain Research and Intervention Center of Excellence

Gainesville, Florida

Joseph T. Hanlon, Pharm.D., M.S.

Professor of Medicine and

Health Scientist

Division of Geriatric Medicine

University of Pittsburgh

Pittsburgh, Pennsylvania 


\section{Working Group Planning Meeting for the National Institutes of Health (NIH) Pathways to Prevention Workshop: Effectiveness and Risks of Long-Term Opioid Treatment of Chronic Pain}

August 28-29, 2013

\section{Working Group}

Christopher M. Jones, Pharm.D., M.P.H.

Lieutinent Commander, U.S. Public Health

Service

Team Lead

Prescription Drug Overdose Team

Division of Unintentional Injury Prevention

National Center for Injury Prevention and Control

Centers for Disease Control and Prevention

Chamblee, Georgia

Margaret Kotz, D.O.

Director

Addiction Recovery Services

Department of Psychiatry-Adult

University Hospitals Case Medical Center

Professor, Psychiatry

Professor, Anesthesiology

Case Western Reserve University School of

Medicine

Cleveland, Ohio

Deborah Langer, M.P.H.

Senior Communications Advisor

Office of Disease Prevention

Division of Program Coordination,

Planning, and Strategic Initiatives

Office of the Director

National Institutes of Health

Bethesda, Maryland

Elinore F. McCance-Katz, M.D., Ph.D.

Chief Medical Officer

Substance Abuse and Mental Health

Services Administration

Rockville, Maryland
David M. Murray, Ph.D.

Associate Director for Prevention

Director

Office of Disease Prevention

Division of Program Coordination,

Planning, and Strategic Initiatives

Office of the Director

National Institutes of Health

Bethesda, Maryland

Elizabeth Neilson, M.S.N., M.P.H.

Senior Communications Advisor

Office of Disease Prevention

Division of Program Coordination,

Planning, and Strategic Initiatives

Office of the Director

National Institutes of Health

Bethesda, Maryland

Ann O'Mara, Ph.D., R.N., FAAN

Head

Palliative Care Research

Community Oncology and Prevention Trials

Research Group

Division of Cancer Prevention

National Cancer Institute

National Institutes of Health

Bethesda, Maryland

Wilma Peterman Cross, M.S.

Deputy Director

Office of Disease Prevention

Division of Program Coordination,

Planning, and Strategic Initiatives

Office of the Director

National Institutes of Health

Bethesda, maryland 


\section{Working Group Planning Meeting for the National Institutes of Health (NIH) Pathways to Prevention Workshop: Effectiveness and Risks of Long-Term Opioid Treatment of Chronic Pain}

August 28-29, 2013

\section{Working Group}

Bob A. Rappaport, M.D.

Director

Division of Anesthesia, Analgesia, and Addiction Products

Center for Drug Evaluation and Research (CDER)

Food and Drug Administration

Silver Spring, Maryland

David B. Reuben, M.D.

Chief

Geriatric Division

Director

Multicampus Program in Geriatric Medicine and Gerontology

Professor of Medicine

Division of Geriatrics

David Geffen School of Medicine at the University of California, Los Angeles

Los Angeles, California

Wendy B. Smith, Ph.D., M.A., B.C.B.

Director for Research Development and Outreach

Office of Behavioral and Social Sciences

Research

Office of the Director

National Institutes of Health

Bethesda, Maryland
Judith Turner, Ph.D.

Professor

Department of Psychiatry and Behavioral Sciences

University of Washington

Seattle, Washington

Michael Von Korff, Sc.D.

Senior Investigator

Group Health Research Institute

Group Health Cooperative

Paris A. Watson

Senior Advisor

Office of Disease Prevention

Division of Program Coordination, Planning, and Strategic Initiatives

Office of the Director

National Institutes of Health

Jessica Wu, Ph.D.

Health Scientist

Office of Disease Prevention

Division of Program Coordination, Planning, and Strategic Initiatives

Office of the Director

National Institutes of Health 


\section{Workshop Sponsors}

NIH Office of Disease Prevention

David M. Murray, Ph.D.

Director

NIH Pain Consortium

Story Landis, Ph.D.

Chair

National Institute on Drug Abuse

Nora D. Volkow, M.D.

Director

National Institute of Neurological Disorders and Stroke

Story Landis, Ph.D.

Director 